\title{
Impact of Tryptophan Depletion on Executive System Function during Menopause is Moderated by Childhood Adversity
}

\author{
Sheila Shanmugan ',2, James Loughead', Wen Cao', Mary D Sammel ${ }^{2,3,4}$, Theodore D Satterthwaite', \\ Kosha Ruparel', Ruben C Gur' and C Neill Epperson*,1,2,4
}

'Department of Psychiatry, Perelman School of Medicine at the University of Pennsylvania, Philadelphia, PA, USA; ${ }^{2}$ Penn PROMOTES Research on Sex and Gender in Health, University of Pennsylvania, Philadelphia, PA, USA; ${ }^{3}$ Department of Biostatistics and Epidemiology, Perelman School of Medicine at the University of Pennsylvania, Philadelphia, PA, USA; ${ }^{4}$ Department of Obstetrics and Gynecology, Perelman School of Medicine at the University of Pennsylvania, Philadelphia, PA, USA

\begin{abstract}
Many healthy women with no history of cognitive dysfunction experience subjective executive difficulties during menopause. Preclinical literature suggests latent effects of early life adversity on serotonin function may play a role in this phenomenon. However, evidence in human participants regarding the mechanisms by which loss of estradiol contributes to this vulnerability is lacking. Here we examined the impact of tryptophan depletion (TD) and adverse childhood experiences (ACE) on brain activation during a working memory task in menopausal women. We hypothesized that an interactive effect between ACE and TD would be observed when women were hypogonadal, and that treatment with estradiol would attenuate this effect. Thirty-three women underwent functional imaging at four time points ( 123 total scans) in this double-blind, placebo controlled, cross-over study. The effects of TD, ACE, and TD $\times$ ACE were evaluated using a voxel-wise, mixed-effects, $2 \times 2$ ANOVA. In the absence of exogenous estradiol, a TD by ACE interaction was observed on BOLD signal in the right DLPFC such that TD increased activation in high ACE subjects but decreased activation in low ACE subjects. While a similar interaction was observed with placebo treatment, treatment with estradiol attenuated the effects of ACE and TD such that no between or within group differences were observed. Together, these results suggest that early life adversity may have a lasting impact on serotonergic circuits underlying executive function that are unmasked by loss of estradiol during menopause. Neuropsychopharmacology (2017) 42, 2398-2406; doi:I0.1038/npp.2017.64; published online 12 April 2017
\end{abstract}

\section{INTRODUCTION}

During menopause, many healthy women experience new onset subjective difficulties with executive processes involving sustained attention, motivation for work, organization and working memory (Epperson et al, 2015; Shanmugan et al, 2017). Onset of these executive difficulties coincides with waning estradiol levels and may be a result of reduced estradiol modulation of the prefrontal cortex (PFC; Shanmugan and Epperson, 2014). We previously showed that estradiol modulation of serotonergic function in the dorsolateral PFC (DLPFC) may be particularly important in maintaining intact working memory processes in menopausal women (Epperson et al, 2012). Early life experiences may also shape an individual's executive function during this hormonal transition (Shanmugan and Epperson, 2014). Significant adversity during childhood alters executive

* Correspondence: Dr CN Epperson, Department of Psychiatry or Department of Obstetrics and Gynecology, Perelman School of Medicine at the University of Pennsylvania, Penn Center for Women's Behavioral Wellness, 3535 Market Street, 3rd Floor, Philadelphia, PA 19104, USA, Tel: +(215) 573-887I, Fax: +(215) 573-888I, E-mail: cepp@mail.med.upenn.edu

Received 22 December 2016; revised 30 January 2017; accepted 5 March 2017; accepted article preview online 21 March 2017 system development (Bruce et al, 2013; Shanmugan and Satterthwaite, 2016), resulting in poor working memory and executive system hyperactivation into adulthood (Bruce $e t a l$, 2013; Majer et al, 2010; Philip et al, 2016) as well as suboptimal cognitive aging (Barnes et al, 2012). Moreover, adverse childhood experiences (ACE) have lasting impacts on serotonergic function (Comasco et al, 2013; van der Doelen et al, 2015), which may leave individuals vulnerable to cognitive changes during periods of low estradiol such as during menopause (Epperson et al, 2011; Epperson et al, 2015; Shanmugan and Epperson, 2014; Shanmugan et al, 2017).

Non-human primate studies indicate effects of early life stress and estradiol converge on key aspects of serotonergic function. Specifically, reduction in tryptophan hydroxylase type 2 (TPH2) gene expression resulting from social subordination stress in ovariectomized macaques is ameliorated by treatment with estradiol (Bethea et al, 2008). In nonstressed ovariectomized rats and macaques, chronic estradiol treatment not only increases serotonin synthesis via TPH2, but also enhances serotonin release and turnover, increases serotonin transporter binding, and increases serotonin receptor 2A mRNA and binding (Amin et al, 2005). Together, this preclinical literature suggests early life stress and estradiol act on common serotonergic targets at the 
molecular level. However, what roles early adversity and the interaction between early adversity and serotonin function play in conferring vulnerability to executive dysfunction during menopause is unknown. Human participants studies examining ACE effects on serotonergic function during conditions of low and high estradiol are needed to determine whether early adversity increases risk for executive dysfunction during menopause via effects on serotonin.

We used tryptophan depletion (TD) and a double-blind, placebo-controlled, cross-over design in 33 healthy menopausal women with high and low levels of early life adversity. We hypothesized that ACE would be associated with aberrant activation in the DLPFC. In addition, we predicted TD would differentially impact DLPFC activation in high $v s$ low ACE hypogonadal women. Moreover, we hypothesized that estradiol treatment would attenuate ACE and TD effects.

\section{MATERIALS AND METHODS}

Participants were healthy women aged 48-60 with no psychiatric diagnoses and within 10 years of their last menstrual period. Participants were right-handed and had a FSH $>30 \mathrm{IU} / \mathrm{ml}$, a normal mammogram within the last year, and a clear urine toxicology. Exclusion criteria included use of hormone therapy within the last year, contraindications to hormone therapy, IQ $<95$, Mini Mental Status Exam Score $<25$, lifetime history of psychotic or bipolar disorder, other psychiatric or substance use disorder in the last year, Hamilton depression score $>14$, psychotropic medication use within the last month, metallic implant, and claustrophobia. This study was approved by the Institutional Review Board at the University of Pennsylvania. All participants provided written informed consent.

\section{Study Design}

Participants underwent 4 test days in this double-blind, placebo-controlled, cross-over study (Figure 1a). During test days 1 and 2 (phase 1), participants underwent 2 imaging sessions: active TD or sham TD. The order of condition was counterbalanced and double-blind. After phase 1, participants were randomized to $17 \beta$-estradiol (Vivelle Dot $0.100 \mathrm{mg} /$ day) or placebo patch treatment for 10 weeks. After completing $\sim 8$ weeks of estradiol or placebo, participants underwent active TD and sham TD (phase 2).

On test days, participants ingested capsules containing either amino acids without tryptophan (active TD) or microcellulose (sham TD). Blood was taken for free tryptophan analysis (Figure $1 \mathrm{~b}$ ) and mood ratings were completed before consumption of capsules and $\sim 6 \mathrm{~h}$ later, after which participants underwent neuroimaging. Subjects with percent change in tryptophan $\sim>3 \mathrm{SD}$ from the average for the assigned depletion status were excluded to ensure that tryptophan levels were consistent with the assigned condition.

\section{Assessment of Early Life Adversity}

The Adverse Childhood Experiences Questionnaire (Felitti et al, 1998) was used to assess history of emotional, physical, or sexual abuse, childhood neglect, and household dysfunction. The ACE questionnaire assesses the number of exposures rather than severity (Teicher and Parigger, 2015) and has been used extensively to assess the association between early adversity and later life health outcomes (Centers for Disease Control and Prevention, 2014a). Number of exposures was summed to create the ACE score (range: $0-10$ ). Subjects with an ACE score $\geqslant 2$ were considered 'high ACE' while subjects with ACE score of $<2$ were considered 'low ACE'. A threshold of 2 was used to define the high ACE group based on studies of depression prevalence in later life that demonstrate increased susceptibility at this level of exposure (Chapman et al, 2004; Epperson et al, in press).

\section{Assessment of Mood}

Mood was measured as a possible confound using the Profile of Mood States. Total score and 'Depression-Dejection' subscore assessed changes in overall mood and depressive symptoms, respectively. Difference in mood score (AM-PM) served as the outcome in regression models used to assess the effects of TD and ACE on mood.

\section{Task Paradigm}

A letter $n$-back task was used to probe executive system function during fMRI. The task included four conditions: 0-back, 1-back, 2-back, and 3-back. Stimuli were 4-letter non-words without vowels presented for $500 \mathrm{~ms}$, followed by an interstimulus interval of $2500 \mathrm{~ms}$. In the 0-back condition, participants responded to a target stimulus. In all other conditions, participants responded to a stimulus ' $n$ ' number of stimuli before it. Each condition consisted of 3 20-trial blocks (60 s; 1:2 target-foil ratio). Visual instructions (9s) preceded each block. Baseline rest periods occurred at the beginning (72s), middle ( $24 \mathrm{~s}$ ), and end (24s) of acquisition. Task duration was $924 \mathrm{~s}$. Equivalent $n$-back tasks with unique stimuli were used for each session and version order was counterbalanced. Performance measures of interest were overall true-positive count, false-positive count, and median true-positive response time.

\section{Image Acquisition and Processing}

Imaging data were acquired on $3 \mathrm{~T}$ Siemens Trio scanner. A magnetization-prepared, rapid acquisition gradient echo T1-weighted image $(\mathrm{TR}=1810 \mathrm{~ms}, \mathrm{TE}=3.51 \mathrm{~ms}, \mathrm{FOV}=$ $180 \times 241 \mathrm{~mm}$, matrix $=192 \times 256,160$ slices, effective voxel resolution of $0.94 \times 0.94 \times 1 \mathrm{~mm}$ ) was acquired to aid spatial normalization to standard space. Functional images were acquired using a whole-brain, single-shot gradient-echo echoplanar sequence with the following parameters: TR/ $\mathrm{TE}=3000 / 32 \mathrm{~ms}, \quad \mathrm{FOV}=192 \times 192 \mathrm{~mm}$, matrix $=64 \times 64$, slice thickness $/ g a p=3 / 0 \mathrm{~mm}, 46$ slices, effective voxel resolution of $3 \times 3 \times 3 \mathrm{~mm}$.

BOLD time series data were skull stripped with BET (Smith, 2002), despiked with AFNI's 3dDespike (Cox, 1996), motion-corrected with MCFLIRT (Jenkinson et al, 2002), high pass filtered $(120 \mathrm{~s})$, spatially smoothed $(6 \mathrm{~mm}$ FWHM), and mean-based intensity normalized. Subjectlevel time series analyses were carried out using FMRIB's Improved Linear Model (FILM) with local autocorrelation correction (Woolrich et al, 2009; Woolrich et al, 2001). The 
a

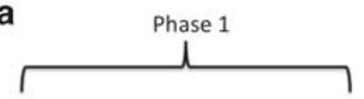

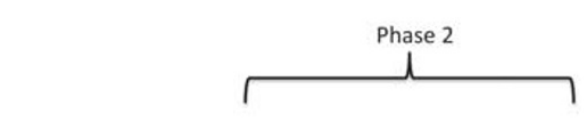

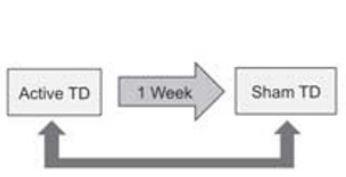

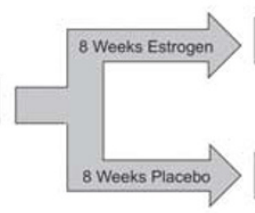

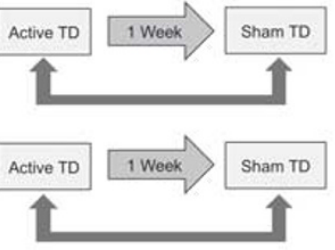

b

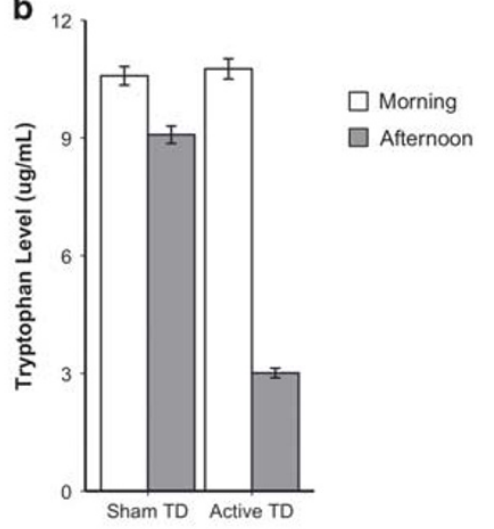

C

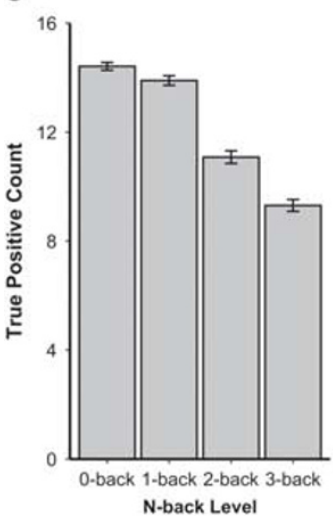

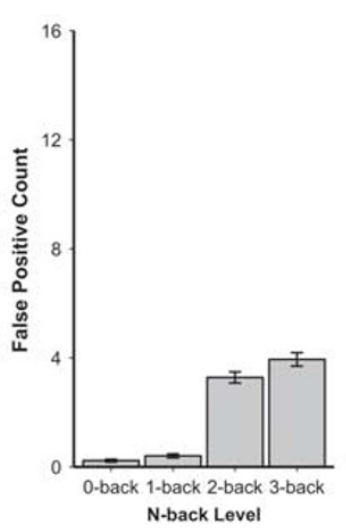

d

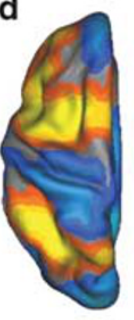

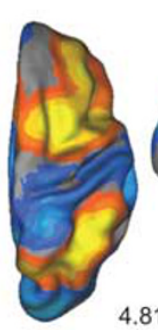

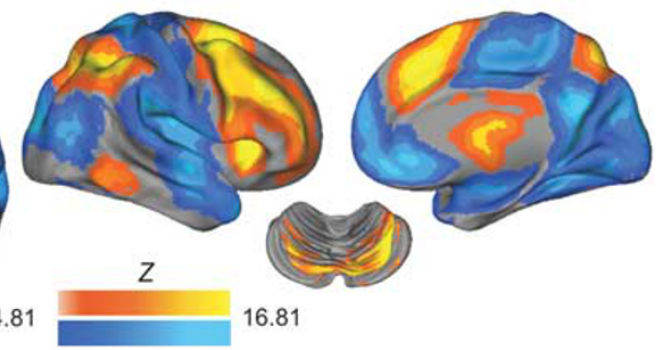

Figure I Study design, tryptophan levels, working memory performance, and contrast of interest. (a) This study used a double-blind, placebo-controlled, cross-over design. Each participant underwent 4 test days. During test days I and 2 (phase I), each participant underwent 2 imaging sessions: active TD or sham TD. The order of condition (active TD vs sham TD) was counterbalanced and double-blind. After completing phase I, participants were randomized to $17 \beta$-estradiol $0.100 \mathrm{mg} /$ day or look-alike placebo patch treatment for a total of 10 weeks. Randomization was assigned by the research pharmacist and was blocked and stratified with a block size of 3 to maintain a 2:I ratio of estrogen to placebo within each stratum. Randomization allocation sequence was generated using a random sequence generator in Excel. Lot and expiration information were withheld by investigational drug services to conceal sequence and randomization status. After completion of $\sim 8$ weeks of estradiol treatment or placebo treatment, each participant again underwent active TD and sham TD on two separate test days (phase 2). On test days, participants presented to the Penn Center for Women's Behavioral Wellness (PCWBW) at 7:30 am after an overnight fast and ingested 70 capsules containing either $31.5 \mathrm{~g}$ of amino acids without tryptophan (active TD) or $31.5 \mathrm{~g}$ of microcellulose (sham TD). The active capsules consisted of L-isoleucine $4.2 \mathrm{~g}$, L-leucine $6.6 \mathrm{~g}$, L-lysine $4.8 \mathrm{~g}$, L-methionine $1.5 \mathrm{~g}$, L-phenylalanine $6.6 \mathrm{~g}$, L-threonine $3.0 \mathrm{~g}$, and L-valine $4.8 \mathrm{~g}$. Enrollment and post-test day follow-up ( $24 \mathrm{~h}$ for placebo, 3 weeks for estradiol) was conducted by research coordinators at PCWBW. (b) Blood was taken for free tryptophan analysis before consumption of the study capsules (morning) and $\sim 6 \mathrm{~h}$ later (afternoon). The effect of active vs sham depletion was assessed using a linear mixed model with percent change in free tryptophan concentration as the outcome. Active TD resulted in a significant decrease in tryptophan level in comparison to sham TD $(p<0.000$ I). There was not a significant difference in percent change in tryptophan levels between ACE groups. Bars represent means and error bars represent standard error. (c) Increased working memory load was associated with fewer correct responses to targets and more false-positive responses to foils. (d) The Bonferonni corrected group mean of the 3-back>0-back contrast across the 123 scans included in analyses activated executive regions and de-activated default mode network regions. Images were displayed using caret. TD, tryptophan depletion.

four condition blocks (0-back, 1-back, 2-back, and 3-back) and their temporal derivatives were modeled using a canonical (double-gamma) hemodynamic response function with standard plus extended motion parameters included as nuisance covariates. The rest condition (fixation point) served as the unmodeled baseline. Functional and anatomical volumes were co-registered using boundary-based registration (Greve and Fischl, 2009). The anatomical image was normalized to the MNI $152 \mathrm{~T} 11 \mathrm{~mm}$ template using diffeomorphic SyN registration in ANTs (Avants et al, 2011; Klein et al, 2009). Co-registration, normalization, and down-sampling to $3 \mathrm{~mm}^{3}$ were concatenated so only one interpolation was performed. Images were assessed for excessive motion (mean relative displacement $>0.5 \mathrm{~mm}$ ) and low temporal signal-to-noise ratio (tSNR $<2 S D$ ).

\section{Group-Level Analyses}

Analyses of imaging data were completed using voxel-wise linear mixed-effects models with FSL's FLAME1 procedure. A two-way, whole-brain, voxel-wise, mixed-effects ANOVA was used to determine the effects of TD, ACE, and TD $\times \mathrm{ACE}$ on brain activation during phase 1 . The primary analysis focused on the parametric contrast; 0-, 1-, 2-, and 3-back were weighted $-3,-1,1$, and 3 to model a linear increase in activity across conditions. The parametric contrast is most sensitive to linear effects in signal associated with increasing working memory load and may not capture non-linear effects. Therefore, we also examined the 3-back $>0$-back contrast. As prior imaging studies found effects of early life stress at 0 -back $>$ baseline (Philip et al, 2016), we also 
examined this contrast. To adequately control for type I error (Eklund et al, 2016) and limit the probability of type 2 error, we implemented stringent cluster correction procedures using 10, 000 Monte-Carlo simulations executed with AFNI's 3dClustSim program (Cox, 1996). Parameters selected include voxel height threshold of $z>3.1$, cluster probability of $p<0.05$, non-Gaussian (long-tailed) filtering, padded simulated volumes to account for edge effect artifacts, 1 -sided thresholding, and first-nearest neighbor clustering (clustering of above threshold voxels if faces touch). Smoothness was estimated with AFNI's 3dFWHMx program using residuals of the group-level analysis and spatial autocorrelation computed as a function of the radius using a mixed Gaussian plus mono-exponential model (Eklund et al, 2016). Mean percent signal change from significant voxels was extracted for further statistical testing and visualization. Signal was also extracted from the 1-back $>$ 0 -back and 2-back $>0$-back contrasts to investigate effects of TD and ACE at varying levels of working memory load (Supplementary Figure S1). To examine whether estradiol attenuated the impact of ACE and TD on BOLD, signal from significant phase 1 voxels was extracted from phase 2 scans.

\section{Statistical Analyses}

Linear mixed-effects models implemented using the nlme package (Pinheiro et al, 2016) in $\mathrm{R}$ ( $\mathrm{R}$ Team, 2015) were

Table I Participant Characteristics at Admission

\begin{tabular}{|c|c|c|}
\hline & Low ACE & High ACE \\
\hline & \multicolumn{2}{|c|}{ Mean (SD) or number (\%) } \\
\hline$n$ & 19 & 14 \\
\hline Age (years) & $55.4(3.6)$ & $54.8(2.9)$ \\
\hline Months since last menstrual period & $52.9(33.4)$ & $62.5(32.1)$ \\
\hline Follicle-stimulating hormone (IU/L) & $93.4(35.6)$ & $75.7(42.8)$ \\
\hline Estradiol $(\mathrm{pg} / \mathrm{mL})$ & $26.5(18.8)$ & $24.1(10.5)$ \\
\hline \multicolumn{3}{|l|}{ Education } \\
\hline Graduate & II (58) & $2(14)$ \\
\hline Some graduate & । (5) & $3(21)$ \\
\hline College & $4(21)$ & $4(29)$ \\
\hline Some college & $2(11)$ & $5(36)$ \\
\hline High school & | (5) & $0(0)$ \\
\hline \multicolumn{3}{|l|}{ Race } \\
\hline Caucasian & $16(84)$ & $9(64)$ \\
\hline African American & $2(11)$ & $4(29)$ \\
\hline Asian & I (5) & I (7) \\
\hline \multicolumn{3}{|l|}{ Employment } \\
\hline Full time & $14(64)$ & $9(64)$ \\
\hline Part time & $\mid(\mid 2)$ & $4(29)$ \\
\hline Retired & $2(\mid 1)$ & $0(0)$ \\
\hline Unknown or preferred not to disclose & $0(0)$ & । (7) \\
\hline
\end{tabular}

employed to evaluate the effect of TD, ACE and their interaction on mood, behavior, and BOLD signal during phase 1 as well as the four-way interaction between ACE group $\times \mathrm{TD}$ status $\times$ estradiol/placebo group $\times$ study phase. All models accounted for repeated measures and controlled for age, estradiol level, and time since last menstrual period. Hypotheses regarding the impact of TD and ACE status under estradiol and placebo conditions on significant phase 1 outcome measures were then tested using this modeling framework. Test day was included as an additional covariate in secondary analyses of significant behavioral findings to account for practice effects. Difference in percent errors between 3-back and 0-back was included as a covariate in supplementary analyses to account for differences in task performance between back levels that may be reflected in the 3-back $>0$-back BOLD contrast. The square root of the coefficient of determination from a linear mixed-effects model was used to estimate the partial correlation between BOLD signal within the DLPFC and behavior while accounting for repeated measures. Spearman's rank correlation was used to examine the relationship between $\mathrm{ACE}$ as a continuous variable and BOLD signal during sham depletion. Statistical tests were two-sided with $p \leqslant 0.05$ considered significant.

\section{RESULTS}

\section{Participants}

Sixty participants completed screening and were enrolled in this study. Two participants self-withdrew before the first test day and 3 were lost to follow-up. Six participants did not complete the first test day due to inability to complete the depletion procedure $(n=1)$, claustrophobia $(n=4)$, and lack of space $(n=1)$ inside the MRI scanner. All study procedures were otherwise well tolerated with no serious or unintended adverse events. Three participants self-withdrew before the second test day, and one subject was withdrawn due to artifacts produced by dental fillings. Per a priori exclusion criteria, participants were excluded from phase 1 analyses for excessive motion $(n=0)$, having a percent change in tryptophan level approximately $>3$ SD from the average for the assigned depletion status $(n=3)$, or missing data regarding ACE $(n=3)$, imaging $(n=1)$, or tryptophan levels $(n=4)$. The subject with the lowest tSNR $(<2 \mathrm{SD})$ was flagged for further inspection; independent component analysis confirmed lack of $n$-back network activation and the subject was excluded.

The final sample included in phase 1 analyses consisted of 19 low ACE and 14 high ACE participants (Table 1, Supplementary Table S1). One of these participants was lost to follow-up before randomization. Only subjects included in phase 1 analyses were considered for inclusion in analyses of phase 2 . Sessions during phase 2 that had percent change in tryptophan level $\sim 3$ SD from the average for each condition $(n=2)$, did not complete imaging $(n=1)$, or had missing tryptophan levels $(n=4)$ were excluded from analyses of phase 2 . The final sample included in phase 2 analyses consisted of 10 low ACE participants (19 sessions) and 11 high ACE participants (19 sessions) randomized to estradiol and 8 low ACE participants (13 sessions) and 3 high ACE participants (6 sessions) randomized to placebo. 


\section{Behavioral Results}

Active TD significantly decreased free tryptophan concentration in comparison to sham TD ( $p<0.0001$; Figure 1b). There was not a significant difference in percent change in tryptophan levels between ACE groups. As expected, increasing working memory load was associated with fewer correct responses to targets and greater false-positive responses to foils (Figure 1c). During phase 1 (prerandomization), active TD increased true-positive responses $(p=0.03)$ in comparison to sham depletion, although had no effect on false-positive responses or reaction time. This effect of TD on true-positive count remained significant $(p=0.04)$ when accounting for non-significant practice effects, although was not present in the smaller subgroups randomized to estradiol or placebo. High ACE was associated with slower true-positive reaction time in comparison to low ACE during phase $1(p=0.1)$, but not in the smaller subgroups of phase 2 . There was no effect of $\mathrm{TD} \times \mathrm{ACE}$ on behavior during either phase of the study. Similarly, there was no significant effect of ACE or TD on overall mood or depressive symptoms.

\section{Imaging Results: Phase 1}

As expected, the parametric (Supplementary Figure S2) and 3 -back $>0$-back (Figure 1d) contrasts robustly recruited the executive network (Loughead et al, 2009; Loughead et al, 2010; Owen et al, 2005; Satterthwaite et al, 2013; Shanmugan et al, 2016). Lower activation during 3-back relative to 0-back in regions associated with the default mode network were also robust. No effects of TD or ACE on the parametric or 0 -back contrasts survived correction, and subsequent analyses focused on the 3-back $>0$-back contrast. A whole-brain analysis demonstrated that TD differentially altered right DLPFC activation in high and low ACE groups (Figure 2a; MNI coordinates: $x=45, y=21, z=48$; volume $=108 \mathrm{~mm}^{3}$; peak $z=3.94)$. BOLD signal in this region significantly correlated with true-positive responses across all subjects $(r=0.34, p=0.007$; Figure 2b). During sham depletion, BOLD signal in this region was significantly correlated with total ACE score $(r=0.41, p=0.02$; Figure $2 c)$. When controlling for the effects of age, estradiol level, and time since last menstrual period, the interaction between ACE and TD remained significant $(p=0.0001)$. Post hoc comparisons revealed the following effects: higher BOLD signal in low ACE participants on active TD compared to sham TD $(p=0.03)$, lower BOLD signal in high ACE participants on active TD compared to sham TD $(p=0.0003)$, and higher BOLD in high ACE participants compared to low ACE participants during sham TD $(p=0.007)$. During active TD, there was no difference between ACE groups. This interactive effect of TD and ACE on DLPFC BOLD remained significant when true positive count was included as an additional covariate $(p=0.0002)$. Similarly, this interaction remained significant $(p=0.0002)$ when controlling for the difference in percent errors between 3-back and 0-back. Post hoc comparisons revealed the following effects: higher BOLD signal in low ACE participants on active TD compared to sham TD $(p=0.03)$, lower BOLD signal in high ACE participants on active TD compared to sham TD $(p=0.0007)$, and higher BOLD in high ACE participants compared to low ACE participants during sham TD $(p=0.005)$. There was no difference between ACE groups during active TD.

\section{Imaging Results: Phase 2}

Within the right DLPFC cluster from phase 1, a four-way interaction between ACE group $\times$ TD status $\times$ estradiol/placebo group $\times$ study phase was detected $(p=0.03)$. A comparable interactive effect to that observed in phase 1 between ACE and TD on BOLD was present in the participants randomized to placebo (Figure $3 \mathrm{~b} ; p=0.07$ ). As in phase 1 , lower BOLD signal was observed in high ACE participants on active TD compared to sham TD $(p=0.08)$ and higher BOLD was observed in high ACE participants compared to low ACE participants during sham TD $(p=0.04)$. However, in participants randomized to estradiol, there was no effect of $\mathrm{ACE}$ or TD on BOLD (Figure 3c). In these participants, estradiol attenuated differences between high and low ACE groups during sham TD as well as BOLD response to active TD in the high ACE group.

Results were similar when controlling for differences in percent errors between 3-back and 0-back. The four-way interaction between ACE group $\times$ TD status $\times$ estradiol/placebo group $\times$ study phase remained significant $(p=0.03)$. An ACE $\times$ TD interaction was observed in participants randomized to placebo $(p=0.09)$; lower BOLD signal was observed in high ACE participants on active TD compared to sham TD $(p=0.1)$ and higher BOLD was observed in high ACE participants compared to low ACE participants during sham TD $(p=0.04)$. No effect of ACE or TD was observed in participants randomized to estradiol.

\section{DISCUSSION}

This double-blind, placebo-controlled, cross-over study examined whether TD differentially impacted brain activation in high vs low ACE hypogonadal women in the presence and absence of exogenous estradiol. In the absence of exogenous estradiol, TD increased right DLPFC BOLD signal and improved working memory performance in women who experienced low levels of early life adversity. In contrast, TD decreased right DLPFC activation without worsening performance in high ACE women. However, treatment with estradiol attenuated the effects of ACE and TD such that no between or within group differences were observed. Together, these results suggest that ACE has lasting impacts on serotonergic circuits supporting executive function that are unmasked by loss of estradiol during menopause.

\section{Evidence for TD $\times$ ACE Effects on Executive Function}

As expected, we found main effects of ACE and TD as well as an ACE by TD interaction on DLPFC activation in hypogonadal women. The DLPFC is part of the frontoparietal network responsible for goal-directed executive functions (Cortese et al, 2012) and dysfunction of this region contributes to executive deficits across multiple psychiatric disorders (Shanmugan et al, 2016). During sham depletion, the high ACE group exhibited significantly greater activation in this region and a trend for slower reaction time than the low ACE group. This finding suggests early adversity is 
a

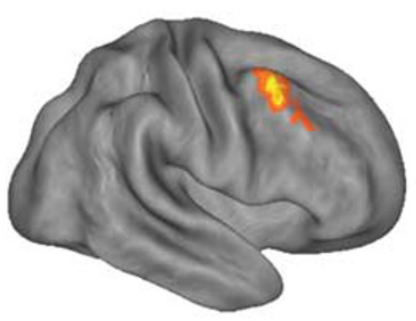

Z 3.1 3.94 b

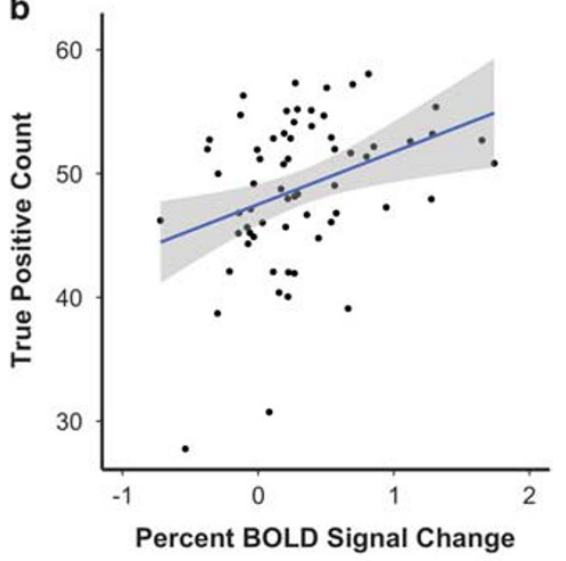

d

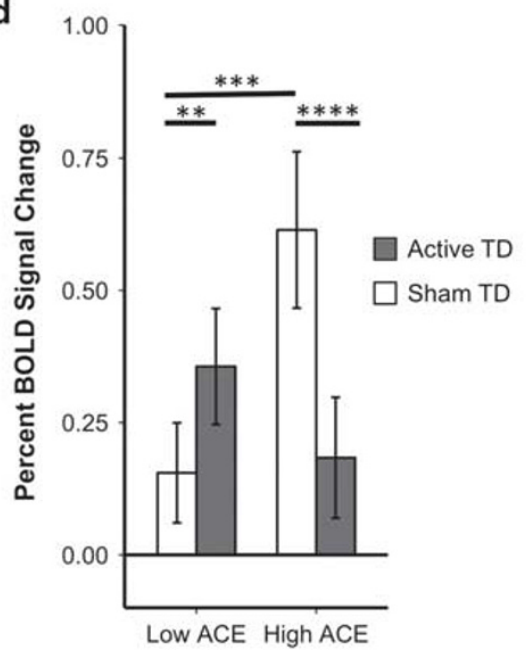

Figure 2 Phase I imaging results. (a) A whole-brain mixed-effects $2 \times 2$ ANOVA demonstrated that TD differentially altered right DLPFC activation in high and low ACE groups (MNI coordinates: $x=45, y=21, z=48$; volume $=108 \mathrm{~mm}^{3}$; peak $z=3.94$ ). (b) BOLD signal in this region significantly correlated with true-positive responses across all subjects $(r=0.34, p=0.007)$. This relationship remained significant when excluding the two sessions with the lowest number of true positive responses $(r=0.30, p=0.02)$. (c) During sham depletion, BOLD signal in this region was significantly correlated with total ACE score ( $r=0.4 \mathrm{I}$, $p=0.02$ ). (d) Signal from the region displayed in Figure 2 a was extracted for visualization. In the low ACE group, active TD increased BOLD relative to sham TD $(\beta=0.20,95 \% \mathrm{Cl}:-0.16-0.21, p=0.03)$. In the high ACE group, active TD decreased activation relative to sham TD $(\beta=-0.43,95 \% \mathrm{Cl}:-0.65$ to $-0.22, p=0.0003)$. During sham depletion, high ACE was associated with greater activation relative to low ACE $(\beta=0.49,95 \%$ Cl: $0.15-0.82, p=0.007)$. During active TD, there was no difference between ACE groups. Bars represent means and error bars represent standard error. ACE, adverse childhood

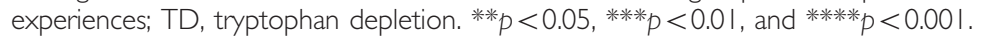

associated with inefficient hyperactivation of the DLPFC in hypogonadal menopausal women. Our findings are globally convergent with the only other study that used a whole-brain approach to examine early adversity effects on brain activation during the $n$-back in healthy adults, which found that early adversity was associated with hyperactivation of several brain regions (Philip et al, 2016). While that study found effects of early life adversity during 0 -back, differences in results between our study and theirs are likely due to differences in correction methods employed and instruments used to assess early adversity.

Although the high ACE group demonstrated inefficient hyperactivation of the DLPFC during sham TD, this ACE effect was not present during active TD. That TD ameliorates this ACE-associated inefficiency in DLPFC activation suggests that serotonergic differences likely contribute to baseline differences in BOLD between ACE groups observed during the sham condition. This finding is in agreement with two other $n$-back studies demonstrating TD-induced decreases in DLPFC activation in the absence of performance changes (Allen et al, 2006; Epperson et al, 2012). In contrast, TD increased activation and task performance in the low ACE group. Similar TD-induced improvements in performance (Booij et al, 2005; Schmitt et al, 2000; Scholes et al, 2007) have been reported during tasks requiring focused attention and response inhibition (Mendelsohn et al, 2009). These studies may not be directly relevant to our results as they examined executive domains other than working memory. The combination of group differences observed during sham depletion and the differential direction of response to active depletion highlight that the effects of ACE should be accounted for in future neuroimaging studies utilizing neurotransmitter manipulations. Failure to account for this moderating effect is likely a major factor contributing to the inconclusive supporting literature regarding TD's effects on executive function. 

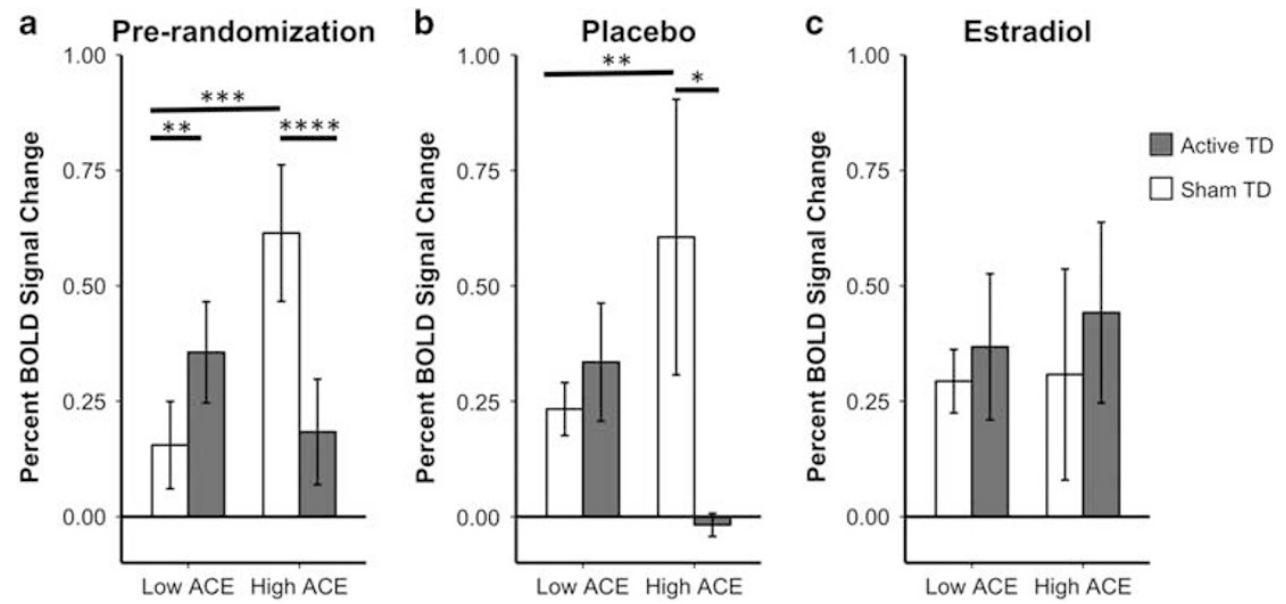

Figure 3 Phase 2 imaging results. (a) TD and ACE exerted an interactive effect on right DLPFC BOLD during phase one. This panel is identical to Figure 2c. (b) Within this cluster, a four-way interaction between ACE group $\times$ TD status $\times$ estradiol/placebo group $\times$ study phase was detected $(\beta=1.1,95 \% \mathrm{Cl}$ : $0.12-2.1, p=0.03)$. A comparable interactive effect to that observed in phase I between ACE and TD on BOLD was present in the participants randomized to placebo. Active TD decreased activation in the high ACE group in comparison to sham TD $(\beta=-0.62,95 \% \mathrm{Cl}:-1.4$ to $0.12, p=0.08)$ and high ACE was associated with increased activation in comparison to low ACE during sham TD ( $\beta=0.59,95 \% \mathrm{Cl}: 0.12-1.1, p=0.04)$. (c) Estradiol attenuated differences between high and low ACE groups during sham TD as well as BOLD response to active TD in the high ACE group. There was no effect of ACE or TD on BOLD after estradiol treatment. Bars represent means and error bars represent standard error. ACE, adverse childhood experiences; TD, tryptophan depletion. $* p<0.1, * * p<0.05$, ${ }^{* * *} p<0.01$, and $* * * * * 0<0.001$.

In combination, these results suggest that ACE has lasting impacts on serotonin function and that hypogonadal middleaged women with high and low levels of early adversity respond differently to experimental manipulations of the serotonergic system. As early adversity negatively impacts serotonin synthesis (Shively et al, 2003), metabolism (Wong et al, 2015), and signaling (Murrough et al, 2011), the mechanism contributing to differential TD response in low and high ACE participants is likely multifold. A human participants study found that low socioeconomic status is associated with lower serotonergic activity (Matthews et al, 2000), suggesting differences in baseline serotonin concentration may play a role. Animal studies demonstrating that early life stress lowers TPH2 expression (Shively et al, 2003) and increases monoamine oxidase-A (Wong et al, 2015) support this hypothesis. However, early adversity also reduces 5-HT1B expression (Murrough et al, 2011) and attenuates 5-HT1A function (Matsuzaki et al, 2009). This suggests alterations in feedback inhibition or receptor sensitivity may also contribute to differences in BOLD response to TD between high and low ACE individuals.

\section{Evidence for Estradiol Modulation of TD $\times$ ACE Effects on Executive Function}

As predicted, treatment with estradiol attenuated ACE and TD effects on BOLD signal in the DLPFC. In contrast to the ACE by TD interaction seen in the absence of exogenous estradiol during phase 1 and participants randomized to placebo, no between or within group differences were observed in participants randomized to estradiol. This finding is in agreement with our previous study demonstrating that estradiol prevented TD-induced alterations in brain activation (Epperson et al, 2012). These data support the hypothesis that early adversity has long-term effects on serotonergic circuits involved in working memory that are revealed by loss of estradiol during menopause. Given that TD results in decreased serotonin synthesis, and the animal literature demonstrating effects of early adversity and estradiol on TPH2 gene expression (Shively et al, 2003), one likely mechanism by which ACE is exerting these latent effects on working memory may be via TPH2. However, the converging effects of early trauma (Matsuzaki et al, 2009) and estradiol (Michopoulos et al, 2014) on 5-HT1A make the presynaptic autoreceptor another promising alternative.

\section{Limitations}

While this study leveraged a unique study design with several levels of rigorous controls, certain limitations merit discussion. First, given the association between ACE and many adverse health-related outcomes (Centers for Disease Control and Prevention, 2014b), the resilient sample of highly educated, physically and psychologically healthy hypogonadal women studied here reduces generalizability to the typical menopausal population, particularly those with substantial early life stress. However, using this sample allowed us to examine the effects of early life adversity on serotonin system function while limiting the potentially confounding impacts of comorbid psychiatric symptomatology and psychotropic medication use. Second, no parametric effects of ACE or TD survived correction, and results are based on a post hoc analysis of the 3-back $>0$-back contrast. However, results using this contrast indicate ACE and TD effects are present when high demands are placed on working memory. Third, the sample size of 33 is relatively small. It is possible that lack of ACE and TD effects in the estradiol group could be driven by diminished power in the sub-sample of phase 1 participants. However, these findings are unlikely due to lack of power given the significant fourway interaction as well as the ACE and ACE $\times$ TD effects observed in the smaller sub-sample randomized to placebo. 
In addition, the double-blind, placebo-controlled, cross-over study design using repeated-measures allowed for decreased variance in estimates of treatment effects and increased power to detect between group differences using $>100 \mathrm{fMRI}$ scans, a comparatively large data set for an imaging study employing a pharmacologic manipulation in a healthy population. Furthermore, the stringent Type 1 error correction method applied to our whole-brain analysis highlights the robust nature of these findings.

\section{CONCLUSIONS}

In summary, these data imply that early life adversity exerts enduring effects on serotonergic circuits underlying executive processes that are unmasked by loss of estradiol during menopause. To confirm this effect of menopause, a longitudinal study of women pre- to postmenopause would be ideal. Our results emphasize serotonin's role in executive function and highlight that the moderating effects of early adversity must be accounted for when manipulating serotonin levels. Further, they provide preliminary mechanistic evidence as to why some, but not all, women experience executive difficulties during menopause and that brain responses to estradiol vary. Further research in a larger sample would be helpful in elucidating the impact of timing of early life adversity on serotonin system function across brain networks, whether sex-differences exist with regard to $\mathrm{TD} \times \mathrm{ACE}$ interactions, and finally whether early life adversity alters the risk-benefit profile of peri- and postmenopausal estrogen treatment.

\section{FUNDING AND DISCLOSURE}

Dr Epperson reports that she has received funding from Shire Pharmaceuticals for investigator initiated research and is site investigator for a multi-site, randomized clinical trial funded by Sage Therapeutics. Dr Epperson also discloses personal investments in the following companies; Pfizer, Johnson and Johnson, Merck, Abbott, and Abbvie. No other authors have any relationships to disclose. This research was supported by P50 MH099910 (Epperson), Penn PROMOTES Research on Sex and Gender in Health (Epperson), R01MH107703 (Satterthwaite), and F30AG055256 (Shanmugan).

\section{ACKNOWLEDGMENTS}

We would like to thank George Anderson, Ph.D. for his measurement of the tryptophan levels.

\section{REFERENCES}

Allen PP, Cleare AJ, Lee F, Fusar-Poli P, Tunstall N, Fu CH et al (2006). Effect of acute tryptophan depletion on pre-frontal engagement. Psychopharmacology 187: 486-497.

Amin Z, Canli T, Epperson CN (2005). Effect of estrogen-serotonin interactions on mood and cognition. Behav Cogn Neurosci Rev 4: $43-58$.

Avants BB, Tustison NJ, Song G, Cook PA, Klein A, Gee JC (2011). A reproducible evaluation of ANTs similarity metric performance in brain image registration. Neuroimage 54: 2033-2044.

Barnes LL, Wilson RS, Everson-Rose SA, Hayward MD, Evans DA, Mendes de Leon CF (2012). Effects of early-life adversity on cognitive decline in older African Americans and whites. Neurology 79: 2321-2327.

Bethea CL, Centeno ML, Cameron JL (2008). Neurobiology of stress-induced reproductive dysfunction in female macaques. $\mathrm{Mol}$ Neurobiol 38: 199-230.

Booij L, Van der Does AJ, Haffmans PM, Riedel WJ, Fekkes D, Blom MJ (2005). The effects of high-dose and low-dose tryptophan depletion on mood and cognitive functions of remitted depressed patients. J Psychopharmacol 19: 267-275.

Bruce J, Fisher PA, Graham AM, Moore WE, Peake SJ, Mannering AM (2013). Patterns of brain activation in foster children and nonmaltreated children during an inhibitory control task. Dev Psychopathol 25(4 Pt 1): 931-941.

Centers for Disease Control and Prevention, National Center for Injury Prevention and Control, Division of Violence Prevention (2014a). About the CDC-Kaiser ACE Study. 2017.

Centers for Disease Control and Prevention, National Center for Injury Prevention and Control, Division of Violence Prevention (2014b). Prevalence of Individual Adverse Childhood Experiences. 2016.

Chapman DP, Whitfield CL, Felitti VJ, Dube SR, Edwards VJ, Anda RF (2004). Adverse childhood experiences and the risk of depressive disorders in adulthood. J Affect Disord 82: 217-225.

Comasco E, Aslund C, Oreland L, Nilsson KW (2013). Three-way interaction effect of 5-HTTLPR, BDNF Val66Met, and childhood adversity on depression: a replication study. Eur Neuropsychopharmacol 23: 1300-1306.

Cortese S, Kelly C, Chabernaud C, Proal E, Di Martino A, Milham MP et al (2012). Toward systems neuroscience of ADHD: a metaanalysis of 55 fMRI studies. Am J Psychiatry 169: 1038-1055.

Cox RW (1996). AFNI: software for analysis and visualization of functional magnetic resonance neuroimages. Comput Biomed Res 29: $162-173$.

Eklund A, Nichols TE, Knutsson H (2016). Cluster failure: why fMRI inferences for spatial extent have inflated falsepositive rates. Proc Natl Acad Sci USA 113: 7900-7905.

Epperson CN, Amin Z, Ruparel K, Gur R, Loughead J (2012). Interactive effects of estrogen and serotonin on brain activation during working memory and affective processing in menopausal women. Psychoneuroendocrinology 37: 372-382.

Epperson CN, Bale TL, Kim DR, Conlin S, Scalice S, Freeman K et al (In Press). Adverse childhood experiences and risk for first episode major depression during the menopause transition. J Clin Psychiatry.

Epperson CN, Pittman B, Czarkowski KA, Bradley J, Quinlan DM, Brown TE (2011). Impact of atomoxetine on subjective attention and memory difficulties in perimenopausal and postmenopausal women. Menopause 18: 542-548.

Epperson CN, Shanmugan S, Kim DR, Mathews S, Czarkowski KA, Bradley J et al (2015). New onset executive function difficulties at menopause: a possible role for lisdexamfetamine. Psychopharmacology 232: 3091-3100.

Felitti VJ, Anda RF, Nordenberg D, Williamson DF, Spitz AM, Edwards V et al (1998). Relationship of childhood abuse and household dysfunction to many of the leading causes of death in adults. The Adverse Childhood Experiences (ACE) Study. Am J Prev Med 14: 245-258.

Greve DN, Fischl B (2009). Accurate and robust brain image alignment using boundary-based registration. Neuroimage 48: 63-72.

Jenkinson M, Bannister P, Brady M, Smith S (2002). Improved optimization for the robust and accurate linear registration and motion correction of brain images. Neuroimage 17: 825-841.

Klein A, Andersson J, Ardekani BA, Ashburner J, Avants B, Chiang MC et al (2009). Evaluation of 14 nonlinear deformation algorithms applied to human brain MRI registration. Neuroimage 46: 786-802.

Loughead J, Ray R, Wileyto EP, Ruparel K, Sanborn P, Siegel S et al (2010). Effects of the alpha4beta2 partial agonist varenicline on 
brain activity and working memory in abstinent smokers. Biol Psychiatry 67: 715-721.

Loughead J, Wileyto EP, Valdez JN, Sanborn P, Tang K, Strasser AA et al (2009). Effect of abstinence challenge on brain function and cognition in smokers differs by COMT genotype. Mol Psychiatry 14: 820-826.

Majer M, Nater UM, Lin JM, Capuron L, Reeves WC (2010). Association of childhood trauma with cognitive function in healthy adults: a pilot study. BMC Neurol 10: 61 .

Matsuzaki H, Izumi T, Matsumoto M, Togashi H, Yamaguchi T, Yoshida $\mathrm{T}$ et al (2009). Early postnatal stress affects 5-HT1A receptor function in the medial prefrontal cortex in adult rats. Eur J Pharmacol 615: 76-82.

Matthews KA, Flory JD, Muldoon MF, Manuck SB (2000). Does socioeconomic status relate to central serotonergic responsivity in healthy adults? Psychosomatic Med 62: 231-237.

Mendelsohn D, Riedel WJ, Sambeth A (2009). Effects of acute tryptophan depletion on memory, attention and executive functions: a systematic review. Neurosci Biobehav Rev 33: 926-952.

Michopoulos V, Perez Diaz M, Embree M, Reding K, Votaw JR, Mun J et al (2014). Oestradiol alters central 5-HT1A receptor binding potential differences related to psychosocial stress but not differences related to 5-HTTLPR genotype in female rhesus monkeys. J Neuroendocrinol 26: 80-88.

Murrough JW, Czermak C, Henry S, Nabulsi N, Gallezot JD, Gueorguieva $\mathrm{R}$ et al (2011). The effect of early trauma exposure on serotonin type $1 \mathrm{~B}$ receptor expression revealed by reduced selective radioligand binding. Arch Gen Psychiatry 68: 892-900.

Owen AM, McMillan KM, Laird AR, Bullmore E (2005). N-back working memory paradigm: a meta-analysis of normative functional neuroimaging studies. Hum Brain Mapp 25: 46-59.

Philip NS, Sweet LH, Tyrka AR, Carpenter SL, Albright SE, Price LH et al (2016). Exposure to childhood trauma is associated with altered n-back activation and performance in healthy adults: implications for a commonly used working memory task. Brain Imaging Behav 10: 124-135.

Pinheiro J, Bates D, DebRoy S, Sarkar D, R Core Team (2016). nlme: Linear and Nonlinear Mixed Effects Models. R Package Version 3.1-126. Available at: http://CRAN.R-project.org/package=nlme.

R Core Team (2015). R: A Language and Environment for Statistical Computing. R Foundation for Statistical Computing: Vienna, Austria.

Satterthwaite TD, Wolf DH, Erus G, Ruparel K, Elliott MA, Gennatas ED et al (2013). Functional maturation of the executive system during adolescence. J Neurosci 33: 16249-16261.

Schmitt JA, Jorissen BL, Sobczak S, van Boxtel MP, Hogervorst E, Deutz NE et al (2000). Tryptophan depletion impairs memory consolidation but improves focussed attention in healthy young volunteers. J Psychopharmacol 14: 21-29.

Scholes KE, Harrison BJ, O'Neill BV, Leung S, Croft RJ, Pipingas A et al (2007). Acute serotonin and dopamine depletion improves attentional control: findings from the stroop task. Neuropsychopharmacology 32: 1600-1610.

Shanmugan S, Epperson CN (2014). Estrogen and the prefrontal cortex: towards a new understanding of estrogen's effects on executive functions in the menopause transition. Human Brain Mapp 35: 847-865.

Shanmugan S, Loughead J, Nanga RP, Elliott M, Hariharan $H$, Appleby D et al (2017). Lisdexamfetamine effects on executive activation and neurochemistry in menopausal women with executive function difficulties. Neuropsychopharmacology 42: 437-445.

Shanmugan S, Satterthwaite TD (2016). Neural markers of the development of executive function: relevance for education. Curr Opin Behav Sci 10: 7-13.

Shanmugan S, Wolf DH, Calkins ME, Moore TM, Ruparel K, Hopson RD et al (2016). Common and dissociable mechanisms of executive system dysfunction across psychiatric disorders in youth. Am J Psychiatry 173: 517-526.

Shively CA, Mirkes SJ, Lu NZ, Henderson JA, Bethea CL (2003). Soy and social stress affect serotonin neurotransmission in primates. Pharmacogenomics J 3: 114-121.

Smith SM (2002). Fast robust automated brain extraction. Human Brain Mapp 17: 143-155.

Teicher MH, Parigger A (2015). The 'Maltreatment and Abuse Chronology of Exposure' (MACE) scale for the retrospective assessment of abuse and neglect during development. PLOS ONE 10: $\mathrm{e} 0117423$.

van der Doelen RH, Arnoldussen IA, Ghareh H, van Och L, Homberg JR, Kozicz T (2015). Early life adversity and serotonin transporter gene variation interact to affect DNA methylation of the corticotropin-releasing factor gene promoter region in the adult rat brain. Dev Psychopathol 27: 123-135.

Wong P, Sze Y, Gray LJ, Chang CC, Cai S, Zhang X (2015). Early life environmental and pharmacological stressors result in persistent dysregulations of the serotonergic system. Front Behav Neurosci 9: 94.

Woolrich MW, Jbabdi S, Patenaude B, Chappell M, Makni S, Behrens $\mathrm{T}$ et al (2009). Bayesian analysis of neuroimaging data in FSL. NeuroImage 45(1 Suppl): S173-S186.

Woolrich MW, Ripley BD, Brady M, Smith SM (2001). Temporal autocorrelation in univariate linear modeling of FMRI data. Neuroimage 14: 1370-1386.

Supplementary Information accompanies the paper on the Neuropsychopharmacology website (http://www.nature.com/npp) 